\title{
ON THE SOLUTIONS OF A STOCHASTIC CONTROL SYSTEM*
}

\author{
TYRONE DUNCAN† AND PRAVIN VARAIYA $\$$
}

\begin{abstract}
The control system considered in this paper is modeled by the stochastic differential equation

$$
d x(t, \omega)=f(t, x(\cdot, \omega), u(t, \omega)) d t+d B(t, \omega),
$$

where $B$ is $n$-dimensional Brownian motion, and the control $u$ is a nonanticipative functional of $x(\cdot, \omega)$ taking its values in a fixed set $U$. Under various conditions on $f$ it is shown that for every admissible control a solution is defined whose law is absolutely continuous with respect to the Wiener measure $\mu$, and the corresponding set of densities on the space $C$ forms a strongly closed, convex subset of $L^{1}(C, \mu)$. Applications of this result to optimal control and two-person, zero-sum differential games are noted. Finally, an example is given which shows that in the case where only some of the components of $x$ are observed, the set of attainable densities is not weakly closed in $L^{1}(C, \mu)$.
\end{abstract}

1. Introduction and contents. A stochastic control problem is defined by the specification of the stochastic differential equation which models the system dynamics, the information available to the controller and the corresponding set of admissible control laws, and the cost incurred by each control law. Of theoretical interest is the "existence" problem, which means determining in terms of the above three defining characteristics a class of control problems for which there exist control laws achieving minimum cost. Published results ([1], [2], [3], see especially the excellent survey article [4] of Fleming) differ from one another and are not usually comparable because either the models are different or the set of admissible control laws is different.

There are two basic steps involved in obtaining an existence result. The first step involves determining conditions which guarantee that a solution of the stochastic differential equation is defined for every admissible control law. The next step involves the search for a topology under which the set of solutions (or an equally good substitute) is compact, and the cost function is lower semicontinuous. Thus, for instance, Fleming and Nisio [1] consider stochastic differential equations of the form

$$
d x(t)=f(t, x(\cdot)) u(t) d t+\sigma(t, x(\cdot), B(\cdot)) d B(t), \quad 0 \leqq t<\infty,
$$

where $u(t)$ is any process taking values in the unit cube, and independent of future increments $B\left(t_{2}\right)-B\left(t_{1}\right), t \leqq t_{1} \leqq t_{2}$, of the Brownian motion $B$. Various conditions on $f, \sigma$ are imposed to guarantee a solution for every admissible control. It is then shown that the set of laws of all the solutions of the differential equation

* Received by the editors August 25, 1970, and in revised form January 11, 1971.

$\uparrow$ Computer Information and Control Engineering Department, University of Michigan, Ann Arbor, Michigan 48104. The work of this author was done while he was visiting the Department of Electrical Engineering and Computer Sciences at the University of California, Berkeley, and was supported by the Office of Naval Research under Contract N0014-67-A-081-0021, NR041-376.

$\$$ Department of Electrical Engineering and Computer Sciences, Electronics Research Laboratory, University of California, Berkeley, California 94720. The work of this author was supported by the U.S. Army Research Office-Durham under Contract DAHC04-67-0046. 
corresponding to the different control laws is compact in the Prohorov metric. Beneš [3] considers stochastic differential equations of the form

$$
d x(t, \omega)=f(t, x(\cdot, \omega), u(t, \omega)) d t+d B(t, \omega), \quad 0 \leqq t \leqq 1,
$$

where $f$ is measurable with respect to its arguments and continuous in $u$. The control law is any nonanticipative, measurable functional $u(t, \omega)=\psi(t, x(\cdot, \omega))$ which takes values in a compact set $U$. He assumes that $f$ satisfies a linear growth condition

$$
|f(t, x(\cdot, \omega), u)|^{2} \leqq K\left(1+\|x(\cdot, \omega)\|^{2}\right),
$$

where $\|x(\cdot, \omega)\|=\sup \{|x(t, \omega)| \quad \mid 0 \leqq t \leqq 1\}$.

The existence of a solution to (1) for every control law is guaranteed by a result of Girsanov [5] (see Corollary 3 below). The resulting law is absolutely continuous with respect to the Wiener measure $\mu$ on the space $C$ of all continuous functions from $[0,1]$ into $R^{n}$. Beneš shows that if $f(t, x(\cdot, \omega), U)$ is convex for every $t \in[0,1]$ and $x(\cdot, \omega) \in C$, then the set of densities corresponding to all the admissible control laws is a convex and strongly closed (hence weakly compact) subset of $L^{1}(C, \mu)$.

In this paper, we show that the above result holds if the linear growth condition is replaced by the growth condition

$$
|f(t, x(\cdot, \omega), u)| \leqq f_{0}(\|x(\cdot, \omega)\|),
$$

where $f_{0}: R \rightarrow R$ is increasing, and the condition

$$
\int_{C} \exp \left[\int_{0}^{1}\langle f(t, B, u), d B(t)\rangle-\frac{1}{2} \int_{0}^{1}|f(t, B, u)|^{2} d t\right] \mu(d B)=1
$$

for every admissible control law. An example is given to show that (2) does not imply (3). The linear growth condition implies (3) (see Corollary 3). Condition (3) also follows from (2), if the drift term $f$ in (1) has a delay (see Corollary 4). Finally we show that in the important case where the control is allowed to depend only on some components of the state $x$, the set of densities is not always weakly closed in $L^{1}(C, \mu)$.

In $\S 2$ we give some preliminary results and definitions, and in $\S 3$ we present the main result on weak compactness of the attainable densities. In $\S 4$ we give conditions which guarantee (3), in $\S 5$ we present applications to optimal control and stochastic differential games, and in the final section we present the negative example for the problem with partial observations.

2. Preliminaries. In the main, we adopt the notations and definitions of Beneš [3]. Consider the stochastic differential equation

$$
\begin{aligned}
d x(t) & =f(t, x, u(t, x)) d t+d B(t), \\
x(0) & =0,
\end{aligned}
$$

where $B(t)$ is a standard $n$-dimensional Brownian motion process with continuous sample paths, $x(t)$ is the state of the system and $u(t, x)$ is the control law which takes values in a compact subset $U$ of $R^{m}$. To state the precise conditions which $f$, $u$ must satisfy we need the following definition. 
Definition 1. (a) Let $C$ be the Banach space of all continuous functions $z:[0,1] \rightarrow R^{n}$ with norm $\|z\|=\max \{|z(t)| \mid 0 \leqq t \leqq 1\}$, where $|y|$ is the Euclidean norm of $y \in R^{n}$.

(b) For each $t \in[0,1]$ let $\mathscr{S}_{t}$ be the smallest $\sigma$-field of subsets of $C$ which contains all sets of the form $\{z \mid z(\tau) \in A\}$, where $\tau \in[0, t]$ and $A$ is a Borel subset of $R^{n}$.

(c) Let $\mathscr{S}=\mathscr{S}_{1}$.

We shall define the solution of (1) in such a way that the sample paths of $x$ are continuous (and have no explosions), so that $f$ is a map from $[0,1] \times C \times U$ $\rightarrow R^{n}$. We impose throughout the following conditions on $f$.

C1. $f$ is measurable with respect to the product $\sigma$-algebra $\mathscr{B} \otimes \mathscr{S} \otimes \mathscr{B}_{U}$, where $\mathscr{B}\left(\mathscr{B}_{U}\right)$ is the set of Borel measurable subsets of $[0,1](U)$.

C2. For fixed $t \in[0,1), f(t, \cdot, \cdot)$ is measurable with respect to the product $\sigma$-algebra $\mathscr{S}_{t} \otimes \mathscr{B}_{U}$.

C3. For fixed $(t, z) \in[0,1] \times C, f(t, z, \cdot)$ is continuous on $U$.

C4. There exists an increasing function $f_{0}: R \rightarrow R$ such that $|f(t, z, u)| \leqq f_{0}(\|z\|)$ for all $(t, z, u)$.

C5. $f(t, z, U)$ is closed and convex for every $(t, z)$.

Definition 2. (a) An admissible control (law) is any map $u:[0,1] \times C \rightarrow U$ which is measurable with respect to $\mathscr{B} \otimes \mathscr{S}$, and for each fixed $t \in[0,1], u(t, \cdot)$ is measurable with respect to $\mathscr{S}_{t}$. Let $\mathscr{U}$ be the set of all admissible control laws.

(b) For each $u \in \mathscr{U}$, the drift corresponding to $u$ is the function $g=g_{u}:[0,1]$ $\times C \rightarrow R^{n}$ defined by

$$
g(t, z)=f(t, z, u(t, z))
$$

Let $\mathscr{G}=\left\{g_{u} \mid u \in \mathscr{U}\right\}$.

(c) For $g \in \mathscr{G}$ and $N \geqq 0$, let $g^{N}:[0,1] \times C \rightarrow R^{n}$ be defined by

$$
g^{N}(t, z)= \begin{cases}g(t, z) & \text { if }|z(\tau)| \leqq N \quad \text { for } \tau \leqq t \\ 0 & \text { otherwise }\end{cases}
$$

Let $\mathscr{G}^{N}=\left\{g^{N} \mid g \in \mathscr{G}\right\}$.

Definition 3. A function $\psi:[0,1] \times C \rightarrow R^{n}$ will be said to be causal if it is $\mathscr{B} \otimes \mathscr{S}$ measurable, and if for each fixed $t \in[0,1], \psi(t, \cdot)$ is measurable with respect to $\mathscr{S}_{t}$.

From [6, Lemmas 1,2] we can obtain the following useful characterization of $\mathscr{G}$. Condition $\mathrm{C} 3$ is needed only for Lemma 1 . The reader should be warned that the proof of the "only if" part of Lemma 1 involves a nontrivial synthesis problem (lemma of Fillipov).

Lemma 1. A causal function $g:[0,1] \times C \rightarrow R^{n}$ belongs to $\mathscr{G}$ if and only if $g(t, z) \in f(t, z, U)$ for all $(t, z)$.

It will prove convenient to work with sets larger than $\mathscr{G}, \mathscr{G}^{N}$.

Definition 4. Let $\Phi$ be the set of all causal maps $\phi:[0,1] \times C \rightarrow R^{n}$ such that $|\phi(t, z)| \leqq f_{0}(\|z\|)$ for all $(t, z)$. Let $\Phi^{N}=\{\phi|\phi \in \Phi,| \phi(t, z) \mid \leqq N$ for all $(t, z)\}$.

Throughout the rest of this paper let $\Omega$ be a fixed space and let $\mathscr{A}_{t}, 0 \leqq t \leqq 1$, be a fixed, increasing family of $\sigma$-fields of subsets of $\Omega$. Let $\mathscr{A}=\mathscr{A}_{1}$. We say that $z(t)$ or $z(t, \omega), 0 \leqq t \leqq 1$, is a family of $n$-dimensional random variables on $\left(\Omega, \mathscr{A}_{t}\right)$ if for each $t, z(t, \cdot)$ is a map from $\Omega$ into $R^{n}$ which is measurable with respect to $\mathscr{A}_{t}$. 
We shall need to consider various probability measures on $\mathscr{A}$. If $z(t), 0 \leqq t \leqq 1$, is a family of $n$-dimensional random variables on $\left(\Omega, \mathscr{A}_{t}\right)$ and we wish to consider the stochastic process generated by $z(t)$ corresponding to a particular probability measure $P$ on $\mathscr{A}$, we will say that $z(t), 0 \leqq t \leqq 1$, is an $n$-dimensional stochastic process on $\left(\Omega, \mathscr{A}_{t}, P\right)$. Finally let $P_{0}$ be a distinguished probability measure on $\mathscr{A}$, and let $x(t, \omega), 0 \leqq t \leqq 1$, be a fixed $n$-dimensional, Brownian motion process on $\left(\Omega, \mathscr{A}_{t}, P_{0}\right)$ with almost all sample paths $x(\cdot, \omega) \in C$. We assume that the $\sigma$-fields $\mathscr{A}_{t}$ are complete with respect to $P_{0}$.

Definition 5. Let $\psi:[0,1] \times C \rightarrow R^{n}$ be a causal function such that

$$
\int_{0}^{1}|\psi(t, z)|^{2} d t<\infty \quad \text { for all } z \in C
$$

Then $\zeta^{t}(\psi), 0 \leqq t \leqq 1$, is the stochastic process on $\left(\Omega, \mathscr{A}_{t}, P_{0}\right)$ with continuous sample paths, defined by

$$
\zeta^{t}(\psi)=\int_{0}^{t}\langle\psi(\tau, x), d x(\tau)\rangle-\frac{1}{2} \int_{0}^{t}|\psi(\tau, x)|^{2} d \tau .
$$

For convenience, let $\zeta(\psi)=\zeta^{1}(\psi)$. (In (5), the first integral is to be interpreted as an Ito stochastic integral.)

The results of this section are immediate consequences of the work of Girsanov $[5]$.

THEOREM 1 (Existence). Let $\psi:[0,1] \times C \rightarrow R^{n}$ be a causal function such that (4) holds.

(i) Then,

$$
\int_{\Omega} \exp [\zeta(\psi)] P_{0}(d \omega) \leqq 1
$$

(ii) Suppose

$$
\int_{\Omega} \exp [\zeta(\psi)] P_{0}(d \omega)=1
$$

and define the probability measure $P_{\psi}$ on $\mathscr{A}$ by

$$
P_{\psi}(A)=\int_{A} \exp [\zeta(\psi)] P_{0}(d \omega), \quad A \in \mathscr{A}
$$

Then the stochastic process $B(t)$ defined on $\left(\Omega, \mathscr{A}_{t}, P_{\psi}\right)$ by

$$
B(t, \omega)=x(t, \omega)-\int_{0}^{t} \psi(\tau, x(\cdot, \omega)) d \tau, \quad 0 \leqq t \leqq 1,
$$

is a Brownian motion.

(iii) If $\psi$ is bounded, then (6) holds.

Proof. Parts (i), (ii) and (iii) are immediate consequences of Lemma 2, Theorem 1 , and Lemma 1, respectively, of [5]. 
Theorem 1 immediately gives us a sufficient condition for the existence of a solution to (1). For, let $u \in \mathscr{U}$, and let $g$ be the drift corresponding to $u$. If

$$
\int_{\Omega} \exp [\zeta(g)] P_{0}(d \omega)=1
$$

then the stochastic process $x(t)$ on $\left(\Omega, \mathscr{A}_{t}, P_{g}\right)$ satisfies the equation

$$
x(t)=\int_{0}^{t} g(\tau, x) d \tau+\text { Brownian motion. }
$$

LeMma 2. Let $\psi \in \Phi$. Let $y(t), 0 \leqq t \leqq 1$, be a stochastic process on $\left(\Omega, \mathscr{A}_{t}, P\right)$ with continuous sample paths, such that the stochastic process $B(t)$ on $\left(\Omega, \mathscr{A}_{t}, P\right)$ defined by

$$
B(t)=y(t)-\int_{0}^{t} \psi(\tau, y) d \tau, \quad 0 \leqq t \leqq 1,
$$

is a Brownian motion. Then, the measure $v$ induced by $y$ on $(C, \mathscr{S})$ is mutually absolutely continuous with respect to the Wiener measure $\mu$, and

$$
\frac{d \mu}{d v}(y)=\exp \left[-\int_{0}^{1}\langle\psi(t, y), d B(t)\rangle-\frac{1}{2} \int_{0}^{1}|\psi(t, y)|^{2} d t\right] .
$$

Proof. Since $|\psi(\cdot, z)| \leqq f_{0}(\|z\|)$, it follows from Lemma 7 of [5], that the measure $\mu$ on $(C, \mathscr{S})$ defined by

$$
\mu(S)=\int_{S} \exp \left[-\int_{0}^{1}\langle\psi, d B\rangle-\frac{1}{2} \int_{0}^{1}|\psi|^{2} d t\right] d v
$$

coincides with the Wiener measure. It is easy to see that

$$
\exp \left[-\int_{0}^{1}\langle\psi, d B\rangle-\frac{1}{2} \int_{0}^{1}|\psi|^{2} d t\right]>0
$$

$v$-almost everywhere. The result follows.

COROLlaRY 1. Let $\psi \in \Phi$, and let $y(t), 0 \leqq t \leqq 1$, satisfy the hypothesis of Lemma 2. Then

$$
\int_{C} \exp \left[\int_{0}^{1}\langle\psi(t, z), d z(t)\rangle-\frac{1}{2} \int_{0}^{1}|\psi(t, z)|^{2} d t\right] \mu(d z)=1 .
$$

Corollary 2. Let $\psi \in \Phi$, and let $y(t), 0 \leqq t \leqq 1$, satisfy the hypothesis of Lemma 2. Then the measure $v$ on $(C, \mathscr{S})$ induced by $y$ is uniquely specified by $\psi$ and is given $b y$

$$
v(S)=\int_{S} \exp \left[\int_{0}^{1}\langle\psi(t, z), d z(t)\rangle-\frac{1}{2} \int_{0}^{1}|\psi(t, z)|^{2} d t\right] \mu(d z) .
$$

Proof. The corollaries follow from (8) and the identity $d B=d y-\psi(t, y) d t$.

\section{Main results.}

Definition 6. For any subset $\Sigma \subset \Phi$, let $\mathscr{D}(\Sigma)$ be the subset of $L^{1}\left(\Omega, \mathscr{A}, P_{0}\right)$ defined by

$$
\mathscr{D}(\Sigma)=\{\exp \zeta(\phi) \mid \phi \in \Sigma\} .
$$


Proposition 1. $\mathscr{D}\left(\Phi^{N}\right)$ is a bounded subset of $L^{2}\left(\Omega, \mathscr{A}, P_{0}\right)$.

Proof. If $\phi \in \Phi^{N}$, then by definition, $|\phi| \leqq N$. By Lemma 1 of [5], it follows that

$$
\int_{\Omega} \exp 2 \zeta^{t}(\phi) P_{0}(d \omega) \leqq \exp t N^{2}
$$

For the rest of this paper let $E_{0}$ denote expectation with respect to the probability measure $P_{0}$. Also if $\gamma \in L^{1}\left(\Omega, \mathscr{A}, P_{0}\right)$, then $E_{0}\left(\gamma \mid \mathscr{A}_{t}\right)$ denotes the conditional expectation of $\gamma$ with respect to $\mathscr{A}_{t}$.

The proofs of Lemmas 3 and 4 are simple modifications of the proofs of Theorems 4 and 3, respectively, of [3]. They are presented here for completeness and because we shall need to refer to parts of the proofs later.

LEMMA 3. $\mathscr{D}\left(\Phi^{N}\right)$ is a closed subset of $L^{2}\left(\Omega, \mathscr{A}, P_{0}\right)$.

Proof. Let $\phi_{n}, n=1,2,3, \cdots$, be a sequence from $\Phi^{N}$ and let $\rho$ be such that

$$
\lim _{n \rightarrow \infty} E_{0}\left|\rho-\exp \zeta\left(\phi_{n}\right)\right|^{2}=0
$$

and

$$
\lim _{n \rightarrow \infty} \exp \zeta\left(\phi_{n}\right)=\rho \quad \text { a.s. } P_{0} .
$$

First of all $\rho>0$ a.s. $P_{0}$. Because, let $A=\{\omega \mid \rho(\omega)=0\}$. Then from (12),

$$
\lim _{n \rightarrow \infty} \zeta\left(\phi_{n}\right)(\omega)=-\infty \quad \text { for } \omega \in A \text {. }
$$

Also,

$$
\zeta\left(\phi_{n}\right)=\int_{0}^{1}\left\langle\phi_{n}(s, x), d x(s)\right\rangle-\frac{1}{2} \int_{0}^{1}\left|\phi_{n}(s, x)\right|^{2} d s
$$

and $\left|\phi_{n}\right| \leqq N$, so that from (13),

$$
\lim _{n \rightarrow \infty} \int_{0}^{1}\left\langle\phi_{n}(s, x), d x(s)\right\rangle=-\infty \quad \text { on } A .
$$

But

$$
E_{0}\left[\int_{0}^{1}\left\langle\phi_{n}, d x(s)\right\rangle\right]^{2}=E_{0} \int_{0}^{1}\left|\phi_{n}(s)\right|^{2} d s \leqq N^{2},
$$

so that $P_{0}(A)=0$. By Ito's representation [7], there is a causal map

$$
\psi:[0,1] \times C \rightarrow R^{n}
$$

with

$$
\int_{0}^{1}|\psi(t, z)|^{2} d t<\infty
$$

for $z$ in $C$, such that

$$
\rho=1+\int_{0}^{1}\langle\psi(t, x), d x(t)\rangle \text { a.s. } P_{0} .
$$


Let

$$
\rho(t)=E_{0}\left(\rho \mid \mathscr{A}_{t}\right)=1+\int_{0}^{t}\langle\psi(s, x), d x(s)\rangle .
$$

Then, by Jensen's inequality,

$$
\int_{0}^{1} E_{0}\left|\rho(t)-\exp \zeta^{t}\left(\phi_{n}\right)\right|^{2} d t \leqq \int_{0}^{1} E_{0}\left|\rho-\exp \zeta\left(\phi_{n}\right)\right|^{2} d t
$$

which converges to zero, so that taking subsequences if necessary we can assume that

$$
\rho(t)=\lim _{n \rightarrow \infty} \exp \zeta^{t}\left(\phi_{n}\right) \quad \text { a.s. } l \otimes P_{0},
$$

where $l$ denotes Lebesgue measure on $[0,1]$. Next, by Ito's differentiation rule,

$$
\exp \zeta\left(\phi_{n}\right)=1+\int_{0}^{1} \exp \zeta^{t}\left(\phi_{n}\right)\left\langle\phi_{n}(t), d x(t)\right\rangle \text { a.s. } P_{0}
$$

so that

$$
E_{0} \int_{0}^{1}\left|\exp \zeta^{t}\left(\phi_{n}\right) \phi_{n}(t)-\psi(t)\right|^{2} d t=E_{0}\left|\exp \zeta\left(\phi_{n}\right)-\rho\right|^{2}
$$

converges to zero, and therefore, taking subsequences if necessary, we can assume that

$$
\psi(t)=\lim _{n \rightarrow \infty} \exp \zeta^{t}\left(\phi_{n}\right) \phi_{n}(t) \quad \text { a.s. } l \otimes P_{0} .
$$

Since $\rho(t)>0$ a.s. $P_{0}$, we see using (14) that

$$
\psi(t) / \rho(t)=\lim _{n \rightarrow \infty} \phi_{n}(t) \quad \text { a.s. } l \otimes P_{0} .
$$

It follows that there is a causal map $\phi:[0,1] \times C \rightarrow R^{n}$,

$$
\phi(t, x(\cdot, \omega))=\lim _{n \rightarrow \infty} \phi_{n}(t, x(\cdot, \omega)) \quad \text { a.s. } l \times P_{0}
$$

and

$$
\rho(t)=1+\int_{0}^{t} \rho(s)\langle\phi(s, x), d x(s)\rangle .
$$

From Ito's differential rule we see that

$$
d(\log \rho(t))=\langle\phi(t), d x(t)\rangle-\frac{1}{2}|\phi(t)|^{2} d t,
$$

and hence,

$$
\rho=\exp \zeta(\phi) .
$$

Because of (15) we can assume that $|\phi| \leqq N$, so that the lemma is proved.

Lemma 4. $\mathscr{D}\left(\Phi^{N}\right)$ is a convex set. rule,

Proof. Let $\phi_{i} \in \Phi^{N}, \lambda_{i} \geqq 0, i=1,2$, with $\lambda_{1}+\lambda_{2}=1$. By Ito's differentiation

$$
d\left[\exp \zeta^{t}\left(\phi_{i}\right)\right]=\exp \zeta^{t}\left(\phi_{i}\right)\left\langle\phi_{i}(t), d x(t)\right\rangle
$$


Define

$$
\rho(t)=\lambda_{1} \exp \zeta^{t}\left(\phi_{1}\right)+\lambda_{2} \exp \zeta^{t}\left(\phi_{2}\right)
$$

Then

$$
d \rho(t)=\lambda_{1} \exp \zeta^{t}\left(\phi_{1}\right)\left\langle\phi_{1}(t), d x(t)\right\rangle+\lambda_{2} \exp \zeta^{t}\left(\phi_{2}\right)\left\langle\phi_{2}(t), d x(t)\right\rangle
$$

which we can rewrite as

$$
d \rho(t)=\rho(t)\langle\phi(t), d x(t)\rangle,
$$

where

$$
\phi(t)=\frac{\lambda_{1} \exp \zeta^{t}\left(\phi_{1}\right)}{\sum_{i=1}^{2} \lambda_{i} \exp \zeta^{t}\left(\phi_{i}\right)} \phi_{1}(t)+\frac{\lambda_{2} \exp \zeta^{t}\left(\phi_{2}\right)}{\sum_{i=1}^{2} \lambda_{i} \exp \zeta^{t}\left(\phi_{i}\right)} \phi_{2}(t) .
$$

Evidently $\phi \in \Phi^{N}$ since $\phi(t, z)$ is a convex combination of $\phi_{1}(t, z)$ and $\phi_{2}(t, z)$. By Ito's differentiation rule from (16) we obtain

$$
d(\log \rho(t))=\langle\phi(t), d x(t)\rangle-\frac{1}{2}|\phi(t)|^{2} d t
$$

and $\rho(0)=1$ from (14) so that integrating (17) yields

$$
\log \rho(t)=\int_{0}^{t}\langle\phi(s), d x(s)\rangle-\frac{1}{2} \int_{0}^{t}|\phi(s)|^{2} d s .
$$

Hence $\rho(1)=\exp \zeta(\phi)$ and the lemma is proved.

We now state our main result and develop the proof through a sequence of lemmas.

THEOREM 2. (i) $\mathscr{D}(\mathscr{G})$ is a convex set.

(ii) Let

$$
\mathscr{G}^{0}=\left\{g \mid g \in \mathscr{G}, E_{0}(\exp \zeta(g))=1\right\} .
$$

Then, $\mathscr{D}\left(\mathscr{G}^{0}\right)$ is a closed and convex subset of $L^{1}\left(\Omega, \mathscr{A}, P_{0}\right)$.

We shall develop the proof through a sequence of lemmas.

LEMMA 5. $\mathscr{D}(\mathscr{G})$ is convex.

Proof. Let $g_{i}(t, z)=f\left(t, z, u_{i}(t, z)\right)$ with $u_{i} \in \mathscr{U}_{i}, i=1,2$, and let $\lambda_{i} \geqq 0$, with $\lambda_{1}+\lambda_{2}=1$. By Ito's differentiation rule,

$$
d\left(\exp \zeta^{t}\left(g_{i}\right)\right)=\exp \zeta^{t}\left(g_{i}\right)\left\langle g_{i}(t), d x(t)\right\rangle, \quad i=1,2 .
$$

Define

$$
\rho(t)=\lambda_{1} \exp \zeta^{t}\left(g_{1}\right)+\lambda_{2} \exp \zeta^{t}\left(g_{2}\right) .
$$

Then if we repeat the proof of Lemma 4 we can conclude that (noting $\rho(0)=1$ )

$$
\rho(1)=\lambda_{1} \exp \zeta\left(g_{1}\right)+\lambda_{2} \exp \zeta\left(g_{2}\right)=\exp \zeta(\phi),
$$

where $\phi(t, z)$ is a convex combination of $g_{1}(t, z)$ and $g_{2}(t, z)$. Since $g_{i}(t, z) \in f(t, z, U)$, and since this set is convex by condition $\mathrm{C} 5$, we see that

$$
\phi(t, z) \in f(t, z, U)
$$

and hence $\phi \in \mathscr{G}$ by Lemma 1 . The lemma is proved. 
LeMma 6. $\mathscr{D}\left(\mathscr{G}^{0}\right)$ is convex.

Proof. The set

$$
\mathscr{R}=\left\{\rho \mid \rho \in L^{1}\left(\Omega, \mathscr{A}, P_{0}\right), \rho \geqq 0, E_{0} \rho=1\right\}
$$

is convex, and

$$
\mathscr{D}\left(\mathscr{G}^{0}\right)=\mathscr{D}(\mathscr{G}) \cap \mathscr{R},
$$

so that the result follows from Lemma 5 .

Next let $g_{n}, n=1,2, \cdots$, be a sequence from $\mathscr{G}^{0}$, and let $\rho$ be in $L^{1}\left(\Omega, \mathscr{A}, P_{0}\right)$ such that

$$
\lim _{n \rightarrow \infty} \exp \zeta\left(g_{n}\right)=\rho \quad \text { a.s. } P_{0} \quad \text { and in } L^{1}\left(\Omega, \mathscr{A}, P_{0}\right) .
$$

For each positive integer $N$, let

$$
g_{n}^{N}(t, z)= \begin{cases}g_{n}(t, z) & \text { if }|z(\tau)| \leqq N \quad \text { for } \tau \leqq t, \\ 0 & \text { otherwise }\end{cases}
$$

and for $N=1,2,3, \cdots$, inductively select subsequences $g_{k}^{N}, k \in K_{N}$, and $\phi^{N} \in \Phi^{N}$ as follows:

For $N=1$, let $g_{k}^{1}, k \in K_{1}$, be a subsequence of $g_{n}^{1}, n=1,2,3, \cdots$, and let $\phi^{1} \in \Phi^{1}$ be such that

$$
\exp \zeta\left(\phi^{1}\right)=\underset{k \in K_{1}}{\mathrm{w}} \cdot \lim _{\lim } \exp \zeta\left(g_{k}^{1}\right)
$$

(Here and in the remainder w. lim means the weak limit in $\operatorname{L-}^{-}\left(\Omega, \mathscr{A}, P_{0}\right)$.) From Lemmas 4 and $5, \mathscr{D}\left(\Phi^{N}\right)$ is a weakly, sequentially compact subset of $L^{2}(\Omega, \mathscr{A}, P)$ and $g_{n}^{N} \in \Phi^{N}$ so that the above selection makes sense.

Suppose $g_{k}^{N}, k \in K_{N}$, and $\phi^{N} \in \Phi^{N}$ are defined. Then let $g_{k}^{N+1}, k \in K_{N+1}$, be a sequence of $g_{k}^{N}, k \in K_{N}$, and let $\phi^{N+1} \in \Phi^{N+1}$ be such that

$$
\exp \zeta\left(\phi^{N+1}\right)=\underset{k \in K_{N+1}}{\mathrm{w}} \lim \exp \zeta\left(g_{k}^{N+1}\right) .
$$

LeMma 7. Let $C_{N}^{t}=\{z|z \in C| z,(\tau) \mid \leqq N$ for $\tau \leqq t\}$. Then for $i \geqq 0$,

$$
\phi^{N+1}(t, z)=\phi^{N}(t, z) \quad \text { for } 0 \leqq t \leqq 1, \quad z \in C_{N}^{1} .
$$

Proof. First of all from

$$
\exp \zeta\left(\phi^{N}\right)=\mathrm{w}_{k \in K_{N}} \lim \exp \zeta\left(g_{k}^{N}\right)
$$

it is immediate that

$$
E_{0}\left(\exp \zeta\left(\phi^{N}\right) \mid \mathscr{A}_{t}\right)=\underset{k \in K_{N}}{\text { w. } \lim _{0}} E_{0}\left(\exp \zeta\left(g_{k}^{N}\right) \mid \mathscr{A}_{t}\right)
$$

Secondly since

$$
E_{0}\left(\zeta\left(\phi^{N}\right)\right)=1, \quad E_{0}\left(\zeta\left(g_{k}^{N}\right)\right)=1
$$

it follows that a.s. $P_{0}$,

$$
\exp \zeta^{t}\left(\phi^{N}\right)=E_{0}\left(\exp \zeta\left(\phi^{N}\right) \mid \mathscr{A}_{t}\right), \quad \exp \zeta^{t}\left(g_{k}^{N}\right)=E_{0}\left(\exp \zeta\left(g_{k}^{N}\right) \mid \mathscr{A}_{t}\right),
$$


and hence,

$$
\exp \zeta^{t}\left(\phi^{N}\right)=\mathrm{w} \cdot \lim _{k \in K_{N}} \exp \zeta^{t}\left(g_{k}^{N}\right)
$$

Next let

$$
\Omega_{N}^{t}=\left\{\omega \mid \omega \in \Omega, x(\cdot, \omega) \in C_{N}^{t}\right\} .
$$

By definition, for $i \geqq 0$,

$$
g_{k}^{N}(\tau, x(\cdot, \omega))=g_{k}^{N+i}(\tau, x(\cdot, \omega)) \quad \text { for } \tau \leqq t, \quad \omega \in \Omega_{N}^{t},
$$

so that from (19) for $i \geqq 0$,

$$
\exp \zeta^{t}\left(\phi^{N+i}\right)(\omega)=\exp \zeta^{t}\left(\phi^{N}\right)(\omega), \quad \tau \leqq t, \quad \omega \in \Omega_{N}^{t} .
$$

The result now follows if we note that

$$
\begin{aligned}
0=\int_{\Omega_{N}^{1}}\left|\exp \zeta\left(\phi^{N+i}\right)-\exp \zeta\left(\phi^{N}\right)\right|^{2} P_{0}(d \omega)= & \int_{\Omega_{N}^{1}}\left[\int_{0}^{1} \exp \zeta^{t}\left(\phi^{N+i}\right)\left\langle\phi^{N+i}(t), d x(t)\right\rangle\right. \\
& \left.-\int_{0}^{1} \exp \zeta^{t}\left(\phi^{N}\right)\left\langle\phi^{N}(t), d x(t)\right\rangle\right]^{2} P_{0}(d \omega) \\
= & \int_{\Omega_{N}^{1}}\left[\int_{0}^{1} \exp 2 \zeta^{t}\left(\phi^{N}\right)\left|\phi^{N+i}(t, x(\cdot, \omega))-\phi^{N}(t, x(\cdot, \omega))\right|^{2} d t\right] P_{0}(d \omega)
\end{aligned}
$$

so that since $\exp \zeta^{t}\left(\phi^{N}\right)>0$ a.s. $P_{0}$, we must have

$$
\int_{\Omega_{N}^{1}}\left[\int_{0}^{1}\left|\phi^{N+i}(t, x(\cdot, \omega))-\phi^{N}(t, x(\cdot, \omega))\right|^{2} d t\right] P_{0}(d \omega)=0,
$$

and the lemma is proved.

Because of Lemma 7 we can define a causal function $\phi:[0,1] \times C \rightarrow R^{n}$ such that

$$
\phi(t, z)=\phi^{N+i}(t, z) \quad \text { for } 0 \leqq t \leqq 1, \quad\|z\| \leqq N, \quad i \geqq 0 .
$$

From the proof of Lemma 8, and from (18) it follows that

$$
\rho=\exp \zeta(\phi) \text { a.s. } P_{0} \text {. }
$$

Lemma 8 completes the proof of Theorem 2.

LEMMA 8.

$$
\phi \in \mathscr{G}
$$

Proof. Because of (20) and Lemma 1 it is enough to show that

$$
\phi^{N}(t, z) \in f(t, z, U) \text { for } 0 \leqq t \leqq 1, \quad\|z\| \leqq N .
$$

Recall that

$$
\exp \zeta\left(\phi^{N}\right)=\mathrm{w}_{k \in K_{1}} \cdot \lim \exp \zeta\left(g_{k}^{N}\right) .
$$

From the properties of weak $L^{2}$-convergence it is known that there is a convex combination of the $\exp \zeta\left(g_{k}^{N}\right)$ which converges to $\exp \zeta\left(\phi^{N}\right)$ in the $L^{2}$-norm topology. 
More precisely, for each $n$, there are nonnegative numbers $\lambda_{1}^{n}, \cdots, \lambda_{n}^{n}$ with $\lambda_{1}^{n}+\cdots+\lambda_{n}^{n}=1$ such that

$$
\lim _{n \rightarrow \infty} E_{0}\left|\exp \zeta\left(\phi^{N}\right)-\sum_{i=1}^{n} \lambda_{i}^{n} \exp \zeta\left(g_{i}^{N}\right)\right|^{2}=0 .
$$

Let

$$
h_{n}(t)=\sum_{i=1}^{n} \lambda_{i}^{n} \exp \zeta\left(g_{i}^{N}\right)
$$

Repeating the proof of Lemma 4, we can conclude that

$$
h_{n}(t)=\exp \zeta^{t}\left(\eta_{n}\right) \quad \text { a.s. } P_{0}
$$

where $\eta_{n}(t, z)$ is a convex combination of $g_{1}^{N}(t, z), \cdots, g_{n}^{N}(t, z)$. In particular, from the convexity of $f(t, z, U)$ and the fact that $g_{i}^{N}(t, z)=g_{i}(t, z) \in f(t, z, U)$ for $\|z\| \leqq N$, it follows that for $\|z\| \leqq N$,

$$
\eta_{n}(t, z) \in f(t, z, U)
$$

Next

$$
\begin{aligned}
E_{0}\left|\exp \zeta\left(\phi^{N}\right)-\exp \zeta\left(\eta_{n}\right)\right|^{2}= & E_{0} \mid \int_{0}^{1} \exp \zeta^{t}\left(\phi^{N}\right)\left\langle\phi^{N}(t), d x(t)\right\rangle \\
& -\left.\int_{0}^{1} \exp \zeta^{t}\left(\eta_{n}\right)\left\langle\eta_{n}(t), d x(t)\right\rangle\right|^{2} \\
= & E_{0} \int_{0}^{1}\left|\exp \zeta^{t}\left(\phi^{N}\right) \phi^{N}(t)-\exp \zeta^{t}\left(\eta_{n}\right) \eta_{n}(t)\right|^{2} d t
\end{aligned}
$$

converges to zero by (22). Taking subsequences if necessary we see that

$$
\exp \zeta^{t}\left(\phi^{N}\right)(\omega) \phi^{N}(t, x(\cdot, \omega))=\lim _{n \rightarrow \infty} \exp \zeta^{t}\left(\eta_{n}\right)(\omega) \eta_{n}(t, x(\cdot, \omega)) \quad \text { a.s. } l \otimes P_{0},
$$

where $l$ denotes Lebesgue measure on $[0,1]$.

Also a.s. $P_{0}$,

$$
\exp \zeta^{t}\left(\phi^{N}\right)=E_{0}\left(\zeta\left(\phi^{N}\right) \mid \mathscr{A}_{t}\right), \quad \exp \zeta^{t}\left(\eta_{n}\right)=E_{0}\left(\zeta\left(\eta_{n}\right) \mid \mathscr{A}_{t}\right),
$$

so that from (22),

$$
\lim _{n \rightarrow \infty} \int_{0}^{1} E_{0}\left|\exp \zeta^{t}\left(\phi^{N}\right)-\exp \zeta^{t}\left(\eta_{n}\right)\right|^{2} d t=0
$$

and hence taking subsequences if necessary, we have

$$
\exp \zeta^{t}\left(\phi^{N}\right)(\omega)=\lim _{n \rightarrow \infty} \exp \zeta^{t}\left(\eta_{n}\right)(\omega) \quad \text { a.s. } l \otimes P_{0} .
$$

Since $\exp \zeta^{t}\left(\phi^{N}\right)>0$ a.s. $P_{0}$, we conclude from (24) that

$$
\phi^{N}(t, x(\cdot, \omega))=\lim _{n \rightarrow \infty} \eta_{n}(t, x(\cdot, \omega)) \quad \text { a.s. } l \otimes P_{0},
$$

and hence from (23), and the fact that $f(t, z, U)$ is closed, we see that

$$
\phi^{N}(t, x(\cdot, \omega)) \in f(t, x(\cdot, \omega), U) \text { for }\|x(\cdot, \omega)\| \leqq N, \quad \text { a.s. } l \otimes P_{0} .
$$


From Corollary 1 of Lemma 2 and from Theorem 2 we obtain Theorem 3. THEOREM 3. Suppose $f$ satisfies C1-C5 of $\S 2$.

(i) For an admissible control $u \in \mathscr{U}$, there exists a solution to (1) with continuous sample paths (without explosions) if and only if

$$
E_{0} \exp \zeta\left(g_{u}\right)=1 \text {. }
$$

(ii) The set of densities $\left\{\exp \zeta\left(g_{u}\right) \mid E_{0} \exp \zeta\left(g_{u}\right)=1\right\}$ is a convex set, which is closed in the norm topology of $L^{1}\left(\Omega, \mathscr{A}, P_{0}\right)$.

4. Sufficient conditions for $E_{0} \exp \zeta(\phi)=1$.

Lemma 9. Let $\phi:[0,1] \times C \rightarrow R^{n}$ be a causal map such that $\int_{0}^{1}|\phi(t, z)|^{2} d t<\infty$ for all $z$ in $C$. Define $T_{\phi}: C \rightarrow C$ by

$$
T_{\phi}(z)(t)=z(t)-\int_{0}^{t} \phi(\tau, z) d \tau .
$$

Suppose that for each $N>0$ there is $M>0$ such that $\left\|T_{\phi}(z)\right\| \leqq N$ implies $\|z\| \leqq M$. Then,

$$
E_{0} \exp \zeta(\phi)=1
$$

Proof. The proof is immediate from Lemma 7 of [5].

As a consequence of Lemma 9, we can obtain the following sufficient conditions. The first result is due to Beneš [3].

COROLlaRY 3. Let $\phi:[0,1] \times C \rightarrow R^{n}$ be a causal map and suppose there is a constant $K$ such that

$$
|\phi(t, z)| \leqq K\left(1+\max _{1 \leqq \tau \leqq t}|z(\tau)|\right) .
$$

Then,

$$
E_{0} \exp \zeta(\phi)=1
$$

Proof. Let $T_{\phi}(z)(t)=y(t)$, and let $\gamma(t)=\max _{0 \leqq \tau \leqq t}|z(\tau)|$. Then, from (25),

$$
\begin{aligned}
\gamma(t) & \leqq|y(t)|+\int_{0}^{t} K(1+\gamma(\tau)) d \tau \\
& \leqq(\|y\|+K)+\int_{0}^{t} K \gamma(\tau) d \tau .
\end{aligned}
$$

By the Bellman-Gronwall inequality,

$$
\begin{aligned}
\|z\|=\gamma(1) & \leqq(\exp K) \gamma(0)+(\exp K)(\|y\|+K) \\
& \leqq(\exp K)(2\|y\|+K),
\end{aligned}
$$

and the result follows from Lemma 9.

The next result is useful if we have a control system with delay.

COROLlaRY 4. Let $\phi:[0,1] \times C \rightarrow R^{n}$ be a causal map such that for some $\delta>0$,

$$
|\phi(t, z)| \leqq f_{0}\left(\max _{0 \leqq \tau \leqq t-\delta}|z(\tau)|\right)
$$


where $f_{0}: R \rightarrow R$ is increasing. Then,

$$
E_{0} \exp \zeta(\phi)=1
$$

Proof. Let $y, \gamma$ be defined as in the previous proof. Then,

$$
\begin{aligned}
\gamma(\delta) & \leqq\|y\|+f_{0}(\gamma(0)), \\
\gamma(2 \delta) & \leqq\|y\|+f_{0}(\gamma(\delta)) \leqq\|y\|+f_{0}\left(\|y\|+f_{0}(\gamma(0))\right) \\
& =f_{1}(\|y\|, \gamma(0)) \quad \text { say. }
\end{aligned}
$$

By induction,

$$
\gamma(i \delta) \leqq f_{i}(\|y\|, \gamma(0)),
$$

where $f_{i}$ is increasing in each argument. Evidently if $(m-1) \delta<1 \leqq m \delta$, we see that

$$
\gamma(1)=\|z\| \leqq f_{m}(\|y\|,|z(0)|),
$$

and the result follows from Lemma 9.

Remark. McKean [8, p. 66] has shown that if $\delta>0$, then all solutions of the one-dimensional diffusion equation

$$
d x(t)=|x|^{1+\delta} d t+d B(t), \quad 0 \leqq t<\infty,
$$

explode with probability 1 . It follows that condition (6) is a nontrivial restriction.

5. Applications. Consider a control system

$$
d x(t, \omega)=f(t, x(\cdot, \omega), u(t, x(\cdot, \omega))) d t+d B(t, \omega),
$$

where the control $u$ takes values in a set $U$ and $f$ obeys the conditions C1-C5 of $\S 2$. Let us impose an additional restriction.

C6. For every admissible $u \in \mathscr{U}$,

$$
E_{0} \exp \zeta\left(g_{u}\right)=1
$$

or equivalently (and directly in terms of $u$ ) for

$$
\rho_{u}(z)=\exp \left[\int_{0}^{1}\langle f(t, z, u(t, z)), d z(t)\rangle-\frac{1}{2} \int_{0}^{1}|f(t, z, u(t, z))|^{2} d t\right]:
$$

C6 $6^{\prime}$.

$$
\int_{C} \rho_{u}(z) \mu(d z)=1
$$

Instead we can limit ourself to the subset $\mathscr{U}^{0}$ consisting of those $u$ in $\mathscr{U}$ which satisfy $\mathrm{C6}^{\prime}$.

Next let $L: C \rightarrow R$ be a bounded function, measurable with respect to $\mathscr{S} . L$ is the cost function and assigns to every $u \in \mathscr{U}^{0}$ the cost

$$
J(u)=\int_{C} L(z) \rho_{u}(z) \mu(d z) .
$$


TheOREM 4. Suppose $\mathscr{U}^{0}$ is nonempty. Then, there exists $u^{*} \in \mathscr{U}^{0}$ such that

$$
J\left(u^{*}\right) \leqq J(u) \text { for all } u \in \mathscr{U}^{0} .
$$

Proof. By Theorem 3, the set $\left\{\rho_{u} \mid u \in \mathscr{U}^{0}\right\}$ is a strongly closed, convex subset of $L^{1}(C, \mathscr{S}, \mu)$. Hence it is weakly compact. Since $\int_{C} L(z) \rho_{u}(z) \mu(d z)$ is linear and continuous in $\rho_{u}$, the result follows.

Let us note that a cost functional of the type (27) allows for a variable endpoint problem as follows. Let $\mathscr{T}$ be a closed subset of $[0,1] \times R^{n}$ which includes the set $\{1\} \times R^{n}$. Let $\lambda:[0,1] \times C \rightarrow R^{n}$ be a bounded, causal function, and to each $u \in \mathscr{U}^{0}$ assign the cost

$$
J(u)=\int_{C}\left[\int_{0}^{t(z)} \lambda(t, z) d t\right] \rho_{u}(z) \mu(d z),
$$

where $t(z)=\min \{\tau \mid z(\tau) \in \mathscr{T}\}$. The term in brackets is clearly of the form $L(z)$ in (27).

If the cost also depends on the control $u$, then sometimes we can add an extra coordinate to the state vector and get an equivalent cost depending only on the state. See [3] for details.

As a second application consider a zero-sum stochastic differential game, with two players I and II, with controls $u_{1}(t) \in U_{1}$ and $u_{2}(t) \in U_{2}$ respectively, and dynamics given by

$$
d x(t)=f\left(t, x, u_{1}(t), u_{2}(t)\right) d t+d B(t) .
$$

Suppose that $f$ splits as

$$
f\left(t, x, u_{1}, u_{2}\right)=\left(\begin{array}{l}
f_{1}\left(t, x, u_{1}\right) \\
f_{2}\left(t, x, u_{2}\right)
\end{array}\right) .
$$

Assume that $f$ satisfies $\mathrm{C} 1-\mathrm{C} 5$ with $\mathrm{C} 5$ now restated as: $f_{1}\left(t, z, U_{1}\right)$ and $f_{2}\left(t, z, U_{2}\right)$ are closed and convex for each $(t, z)$. As before, we define the admissible controls for player $i$, as all causal maps $u_{i}:[0,1] \times C \rightarrow U_{i}, i=1,2$. Let $\mathscr{U}_{i}^{0}$ consist of those admissible controls $u_{i}$ which satisfy

$$
\int_{C} \rho_{u_{i}}^{i}(z) \mu(d z)=1
$$

where

$$
\rho_{u_{i}}^{i}=\exp \left[\int_{0}^{1}\left\langle f_{i}\left(t, z, u_{i}(t, z)\right), d z_{i}(t)\right\rangle-\frac{1}{2} \int_{0}^{1}\left|f_{i}\left(t, z, u_{i}(t, z)\right)\right|^{2} d t\right] .
$$

Here we have split $z=\left(\begin{array}{l}z_{1} \\ z_{2}\end{array}\right)$ to be compatible with $f=\left(\begin{array}{l}f_{1} \\ f_{2}\end{array}\right)$. Let $L: C \rightarrow R$ be a bounded function, measurable with respect to $\mathscr{S}$, and to each pair $\left(u_{1}, u_{2}\right) \in \mathscr{U}_{1}^{0}$ $\times \mathscr{U}_{2}^{0}$ assign to player I the payoff

$$
J\left(u_{1}, u_{2}\right)=\int_{C} L(z) \rho_{\left(u_{1}, u_{2}\right)}(z) \mu(d z)
$$


TheOrem 5. Suppose $\mathscr{U}_{i}^{0}$ is nonempty for $i=1,2$. Then, there exist $u_{i}^{*} \in \mathscr{U}_{i}^{0}$, $i=1,2$, such that

$$
J\left(u_{1}, u_{2}^{*}\right) \leqq J\left(u_{1}^{*}, u_{2}^{*}\right) \leqq J\left(u_{1}^{*}, u_{2}\right) \quad \text { for all } u_{i} \in \mathscr{U}_{i}^{0}, \quad i=1,2 .
$$

Proof. From the definition (26) of $\rho_{\left(u_{1}, u_{2}\right)}$ and the definition (28) we see that

$$
J\left(u_{1}, u_{2}\right)=\int_{C} L(z) \rho_{u_{1}}^{1}(z) \rho_{u_{2}}^{2}(z) \mu(d z)
$$

Next, from Theorem 3 , the sets $\left\{\rho_{u_{i}}^{i} \mid u_{i} \in \mathscr{U}_{i}^{0}\right\}$ are convex, closed subsets of $L^{1}(C, \mathscr{S}, \mu)$, hence weakly compact. Finally the integral in (30) is concave (in fact linear) and continuous in $\rho_{u_{1}}^{1}$ for fixed $\rho_{u_{2}}^{2}$, and convex (in fact linear) and continuous in $\rho_{u_{2}}^{2}$ for fixed $\rho_{u_{1}}^{1}$. Hence from the well-known results on two-person zero-sum games the existence of a saddle point $\left(u_{1}^{*}, u_{2}^{*}\right)$ follows.

6. Partial observations: A negative example. Again consider the stochastic differential equation

$$
d x(t)=f(t, x, u) d t+d B(t) .
$$

The conditions on $f$ are as before, but now suppose that we consider the important case where the control $u$ can only depend upon the past history of the last $m$ $(m<n)$ components of $x$. More precisely, let $Q_{t}$ be the sub- $\sigma$-algebra of $\mathscr{T}_{t}$ generated by all sets of the form

$$
\left\{z \mid z \in C, z_{i}(\tau) \in A\right\}
$$

where $\tau \leqq t, A$ is a Borel subset of $R$ and $n-m+1 \leqq i \leqq n$. Let $\mathscr{U}_{m}$ be the set of all causal maps $u:[0,1] \times C \rightarrow U$ such that $u(t, \cdot)$ is measurable with respect to $Q_{t}$. Evidently,

$$
\mathscr{U}_{m} \subset \mathscr{U}=\mathscr{U}_{n} \text {. }
$$

First of all it should be clear from the proof of Lemma 4 that the set $\left\{\exp \zeta\left(g_{u}\right) \mid u \in \mathscr{U}_{m}\right\}$ may fail to be convex. For, consider the two-dimensional system

$$
d x_{1}=u d t+d B_{1}, \quad d x_{2}=d B_{2},
$$

where the control $u$ is allowed to depend only on $x_{2}$ and must take values in the set $U=[-1,1]$. Now let $u_{1}$ and $u_{2}$ be control laws defined as follows:

$$
\begin{aligned}
& u_{1}\left(t, x_{2}\right) \equiv 0, \\
& u_{2}\left(t, x_{2}\right)= \begin{cases}0, & 0 \leqq t<\frac{1}{2}, \\
\operatorname{sgn}\left(x_{2}\left(\frac{1}{2}\right)\right), & \frac{1}{2} \leqq t \leqq 1\end{cases}
\end{aligned}
$$

It is easy to calculate that

$$
\zeta^{t}\left(g_{u_{1}}\right) \equiv 0
$$

so that $\exp \zeta^{t}\left(g_{u_{1}}\right) \equiv 1$ and

$$
\dot{\zeta}^{t}\left(g_{u_{2}}\right)= \begin{cases}0, & 0 \leqq t<\frac{1}{2}, \\ \operatorname{sgn}\left(x_{2}\left(\frac{1}{2}\right)\right)\left(x_{1}(t)-x_{1}\left(\frac{1}{2}\right)\right)-\frac{1}{2}\left(t-\frac{1}{2}\right), & t \geqq \frac{1}{2}\end{cases}
$$


From the proof of Lemma 4 we know that the function $u_{\lambda}$, given by $u_{\lambda}(t) \equiv 0$, $0 \leqq t \leqq \frac{1}{2}$, and

$$
u_{\lambda}(t)=\frac{\lambda \exp \zeta^{t}\left(g_{u_{2}}\right)}{\lambda \exp \zeta^{t}\left(g_{u_{2}}\right)+(1-\lambda)} u_{2}(t), \quad \frac{1}{2} \leqq t \leqq 1
$$

satisfies

$$
\exp \zeta^{t}\left(g_{u_{\lambda}}\right) \equiv(1-\lambda) \exp \zeta^{t}\left(g_{u_{1}}\right)+\lambda \exp \zeta^{t}\left(g_{u_{2}}\right) .
$$

Since $d \exp \zeta^{t}\left(g_{u_{\lambda}}\right)=\exp \zeta^{t}\left(g_{u_{\lambda}}\right)\left\langle g_{u_{\lambda}}(t), d x(t)\right\rangle, g_{u_{\lambda}}$ (and hence $u_{\lambda}$ ) is uniquely defined by the previous equation. Since for $0<\lambda<1, u_{\lambda}$ depends on $x_{1}$, the assertion is proved.

Next, from Theorem 3 we see that the set $\left\{\exp \zeta\left(g_{u}\right) \mid u \in \mathscr{U}_{m}\right\}$ is weakly compact in $L^{1}\left(\Omega, \mathscr{A}, P_{0}\right)$ if and only if it is weakly closed. We give a simple example to show that in general we do not have weak closure.

Consider the two-dimensional system $x=\left(x_{1}, x_{2}\right)$, with $u \in R$ depending only on $x_{2}$,

$$
d x_{1}(t)=f\left(t, x_{1}\right) u+d B_{1}(t), \quad d x_{2}(t)=d B_{2}(t)
$$

where

$$
f\left(t, x_{1}\right)= \begin{cases}0, & t \leqq \frac{1}{2}, \\ 2, & t>\frac{1}{2}, \quad x_{1}\left(\frac{1}{2}\right)>0 \\ 1, & t>\frac{1}{2}, \quad x_{1}\left(\frac{1}{2}\right) \leqq 0 .\end{cases}
$$

The control set is $U=[-1,1]$. We shall define a sequence of control laws $u_{n}\left(t, x_{2}\right)$ such that

$$
u_{n}\left(t, x_{2}\right)= \begin{cases}0, & t \leqq \frac{1}{2} \\ \gamma_{n}\left(x_{2}\left(\frac{1}{2}\right)\right), & t>\frac{1}{2}\end{cases}
$$

where the functions $\gamma_{n}$ are defined later. It follows that

$$
\begin{aligned}
\zeta_{n} & =\zeta\left(g_{u_{n}}\right)=\int_{0}^{1} f\left(t, x_{1}\right) u_{n}(t) d x_{1}(t)-\frac{1}{2} \int_{0}^{1} f^{2}\left(t, x_{1}\right) u_{n}^{2}(t) d t \\
& =\alpha \beta \gamma_{n}-\frac{1}{4} \beta^{2} \gamma_{n}^{2}
\end{aligned}
$$

where

$$
\alpha=x_{1}(1)-x_{1}\left(\frac{1}{2}\right), \quad \beta= \begin{cases}2 & \text { if } x_{1}\left(\frac{1}{2}\right)>0 \\ 1 & \text { if } x_{1}\left(\frac{1}{2}\right) \leqq 0\end{cases}
$$

Therefore,

$$
\exp \zeta_{n}=\exp \left(\alpha \beta \gamma_{n}\right) \exp \left(-\frac{1}{4} \beta^{2} \gamma_{n}^{2}\right)
$$

We shall select $\gamma_{n}$ such that $\left|\gamma_{n}\right| \equiv 1$, so that (31) simplifies to

$$
\exp \zeta_{n}=\exp \left(\alpha \beta \gamma_{n}\right) \exp \left(-\frac{1}{4} \beta^{2}\right) .
$$

We define $\gamma_{n}$ as follows : 
Let $\xi: R \rightarrow R$ be a measurable function such that under $P_{0}, \xi\left(x_{2}\left(\frac{1}{2}\right)\right)$ is uniformly distributed over $[0,1]$. For each integer $n \geqq 0$, define $\eta_{n}:[0,1] \rightarrow\{-1,1\}$ by

$$
\eta_{n}(\xi)=\left\{\begin{array}{rll}
1 & \text { if } \frac{2 m}{2 n} \leqq \xi<\frac{2 m+1}{2 n}, & m=0,1, \cdots, n-1 \\
-1 & \text { if } \frac{2 m+1}{2 n} \leqq \xi<\frac{2 m+2}{2 n}, & m=0,1, \cdots, n-1
\end{array}\right.
$$

Finally, let

$$
\gamma_{n}\left(x_{2}\left(\frac{1}{2}\right)\right)=\eta_{n}\left(\xi\left(x_{2}\left(\frac{1}{2}\right)\right)\right)
$$

LEMMA 10. $\exp \zeta_{n}$ converges to $\frac{1}{2}[\exp (\alpha \beta)+\exp (-\alpha \beta)] \exp \left(-\frac{1}{4} \beta^{2}\right)$ in the weak topology of $L^{1}\left(\Omega, \mathscr{A}, P_{0}\right)$.

Proof. Let $A_{\alpha}, A_{\beta}, A_{\xi}$ be Borel subsets of $R$ and let $I_{A}$ denote the indicator function of a set $A$. Let

$$
\Pi_{n}=\int_{\Omega} I_{A_{\alpha}}(\alpha(\omega)) I_{A_{\beta}}(\beta(\omega)) I_{A_{\xi}}(\xi(\omega))\left(\exp \zeta_{n}\right)(\omega) P_{0}(d \omega)
$$

Now under $P_{0}$ the random variables $\alpha, \beta, \xi$ are independent, so that

$$
\begin{array}{r}
\Pi_{n}=\int_{-\infty}^{\infty} \int_{-\infty}^{\infty} \int_{-\infty}^{\infty} I_{A_{\alpha}}(\alpha) I_{A_{\beta}}(\beta) I_{A_{\xi}}(\xi) \exp \left(\alpha \beta \eta_{n}(\xi)\right) \exp \left(-\frac{1}{4} \beta^{2}\right) \\
P_{\xi}(d \xi) P_{\beta}(d \beta) P_{\alpha}(d \alpha),
\end{array}
$$

where $P_{\alpha}, P_{\beta}, P_{\xi}$ are the marginal distributions of $\alpha, \beta, \xi$ respectively. From the way $\eta_{n}$ is defined and the fact that $\xi$ is uniformly distributed on $[0,1]$ it follows that for fixed $\alpha, \beta$,

$$
\begin{gathered}
\lim _{n \rightarrow \infty} \int_{-\infty}^{t} \exp \left(\alpha \beta \eta_{n}(\xi)\right) \exp \left(-\frac{1}{4} \beta^{2}\right) P_{\xi}(d \xi) \\
=\int_{-\infty}^{t} \frac{1}{2}[\exp (\alpha \beta)+\exp (-\alpha \beta)] \exp \left(-\frac{1}{4} \beta^{2}\right) P_{\xi}(d \xi)
\end{gathered}
$$

uniformly for $t \in(-\infty, \infty)$. It follows that $\exp \left(\alpha \beta \eta_{n}(\xi)\right) \exp \left(-\frac{1}{4} \beta^{2}\right)$ converges to $\frac{1}{2}[\exp (\alpha \beta)+\exp (-\alpha \beta)] \exp \left(-\frac{1}{4} \beta^{2}\right)$ weakly in $L^{1}\left(R, P_{\xi}\right)$. Since the integrands in (33) are uniformly integrable, it follows that

$$
\begin{aligned}
& \lim _{n \rightarrow \infty} \Pi_{n}=\int_{R^{3}} I_{A_{\alpha}}(\alpha) I_{A_{\beta}}(\beta) I_{A_{\xi}}(\xi) \frac{1}{2}[\exp (\alpha \beta)+\exp (-\alpha \beta)] \\
& \cdot \exp \left(-\frac{1}{4} \beta^{2}\right) P_{\xi}(d \xi) P_{\beta}(d \beta) P_{\alpha}(d \alpha) .
\end{aligned}
$$

From this it follows easily that $\exp \left(\alpha \beta \eta_{n}(\xi)\right) \exp \left(-\frac{1}{4} \beta^{2}\right)$ converges to $\frac{1}{2}[\exp (\alpha \beta)$ $+\exp (-\alpha \beta)] \exp \left(-\frac{1}{4} \beta^{2}\right)$ weakly in $L^{1}\left(R^{3}, P_{\alpha} \otimes P_{\beta} \otimes P_{\xi}\right)$ and the lemma is proved. 
Next, by direct calculation we can show that the two-dimensional drift $\hat{g}$, where $\hat{g}(t)=\left(\begin{array}{l}0 \\ 0\end{array}\right), 0 \leqq t \leqq \frac{1}{2}$, and

$$
\hat{g}(t)=\left(\begin{array}{c}
\beta \frac{\exp 2\left(x_{1}(t)-x_{1}\left(\frac{1}{2}\right)\right)-1}{\exp 2\left(x_{1}(t)-x_{1}\left(\frac{1}{2}\right)\right)+1} \\
0
\end{array}\right), \quad \frac{1}{2} \leqq t \leqq 1
$$

satisfies

$$
\exp \zeta(\hat{g})=\frac{1}{2}[\exp (\alpha \beta)+\exp (-\alpha \beta)] \exp \left(-\frac{1}{4} \beta^{2}\right) .
$$

If we define $\rho(t)=E_{0}\left(\exp \zeta(\hat{\mathrm{g}}) \mid \mathscr{A}_{t}\right)$, then

$$
d \rho(t)=\rho(t)\langle\hat{g}(t), d x(t)\rangle, \quad 0 \leqq t \leqq 1,
$$

so that (34) characterizes $\hat{g}$ uniquely. Hence any control law $\hat{u}$ such that $\exp \zeta\left(g_{u}\right)=\exp \zeta(\hat{g})$ must satisfy $g_{u}=\hat{g}$ so that $\hat{u}$ must depend on $x_{1}$. Therefore the set of densities $\exp \zeta\left(g_{u}\right)$ with $u$ depending only on $x_{2}$ is not weakly closed in $L^{1}\left(\Omega, \mathscr{A}, P_{0}\right)$.

Incidentally this example also shows that to guarantee weak closure, the convexity condition C5 is necessary, for even though $u_{n}(t) \in\{-1,1,0\}$ for all $t$, it is not the case for $\hat{u}(t)$.

Acknowledgments. It should be obvious to anyone who has read [3] our great debt to V. E. Beneš. E. Wong clarified many subtle points about Ito's calculus and suggested the proofs in $\S 2$. M. Davis caught many errors in earlier proofs. It is a pleasure to acknowledge our gratitude to them.

\section{REFERENCES}

[1] W. H. Fleming AND M. Nisio, On the existence of optimal stochastic controls, J. Math. Mech., 15 (1966), pp. 777-794.

[2] H. J. KUSHNER, On the existence of optimal stochastic controls, this Journal, 3 (1965), pp. $463-474$.

[3] V. E. BENEŠ, Existence of optimal stochastic control laws, to appear.

[4] W. H. Fleming, Optimal continuous-parameter stochastic control, SIAM Rev., 11 (1969), pp. 470-509.

[5] I. V. Girsanov, On transforming a certain class of stochastic processes by absolutely continuous substitution of measures, Theor. Probability Appl., 5 (1960), pp. 285-301.

[6] V. E. BENEక̌, Existence of optimal strategies based on specified information, for a class of stochastic decision problems, this Journal, 8 (1970), pp. 179-188.

[7] K. ITo, Multiple Wiener integral, J. Math. Soc. Japan, 3 (1951), pp. 158-161.

[8] H. P. McKean, JR., Stochastic Integrals, Academic Press, New York and London, 1969. 